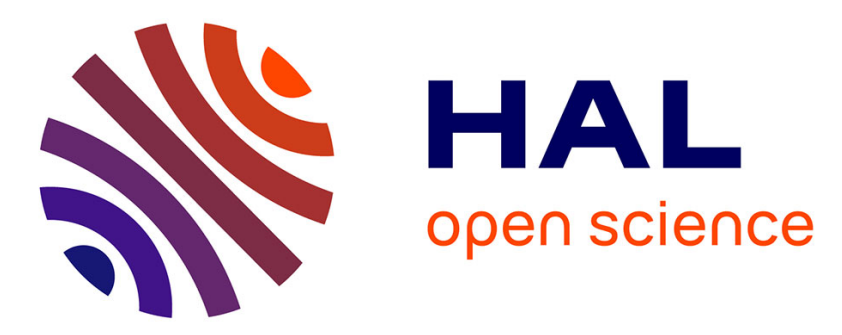

\title{
One-Pot Chemoselective Synthesis of 2,4,6,8-Tetrasubstituted Quinazolines via Microwave-Assisted Consecutive Bis-SNAr/Bis-Suzuki-Miyaura Cross-Coupling Reactions
}

Youssef Kabri, Maxime D. Crozet, Sébastien Redon, Patrice Vanelle

\section{To cite this version:}

Youssef Kabri, Maxime D. Crozet, Sébastien Redon, Patrice Vanelle. One-Pot Chemoselective Synthesis of 2,4,6,8-Tetrasubstituted Quinazolines via Microwave-Assisted Consecutive Bis-SNAr/BisSuzuki-Miyaura Cross-Coupling Reactions. Synthesis: Journal of Synthetic Organic Chemistry, 2017, 10.1055/s-0033-1341105 . hal-01665391

\section{HAL Id: hal-01665391 https://hal.science/hal-01665391}

Submitted on 18 Dec 2017

HAL is a multi-disciplinary open access archive for the deposit and dissemination of scientific research documents, whether they are published or not. The documents may come from teaching and research institutions in France or abroad, or from public or private research centers.
L'archive ouverte pluridisciplinaire HAL, est destinée au dépôt et à la diffusion de documents scientifiques de niveau recherche, publiés ou non, émanant des établissements d'enseignement et de recherche français ou étrangers, des laboratoires publics ou privés. 


\title{
One-pot chemoselective synthesis of 2,4,6,8-tetrasubstituted quinazolines via microwave-assisted consecutive bis-S $\mathrm{S}_{\mathrm{N}} \mathrm{Ar} / \mathrm{bis}-\mathrm{Suzuki}$-Miyaura cross-coupling reactions
}

\author{
Youssef Kabri, Maxime D. Crozet, Sébastien Redon, Patrice Vanelle
}

Aix-Marseille Université, Institut de Chimie Radicalaire ICR, UMR CNRS 7273, Laboratoire de Pharmaco-Chimie Radicalaire, Faculté de Pharmacie, 27 Boulevard Jean Moulin - CS 30064, 13385 Marseille Cedex 05, France

Fax: +33(4)91794677

E-mail: patrice.vanelle@univ-amu.fr

Received: The date will be inserted once the manuscript is accepted.

\begin{abstract}
A practical and efficient synthesis of 2,4,6,8tetrasubstituted quinazolines through one-pot chemoselective sequential bis-S $\mathrm{N}$ Ar/bis-Suzuki-Miyaura reactions under microwave irradiation is presented. The bis- $\mathrm{S}_{\mathrm{N}} \mathrm{Ar}$ occurs selectively at C-2 and C-4 positions of 6,8-dibromo-2,4dichloroquinazoline $\mathbf{4}$ and the bis-Suzuki-Miyaura crosscoupling at C-6 and C-8 positions of 4 . This procedure affords convergent synthesis of polysubstituted quinazoline derivatives in high yields in a very few steps and tolerates a wide range of boronic acids and amines.
\end{abstract}

Key words: quinazoline, Suzuki-Miyaura, chemoselectivity, microwave irradiation, palladium.

The palladium catalyzed Suzuki-Miyaura reaction is one of the most highly regarded processes in synthetic chemistry and a widely studied cross- coupling reaction for carbon-carbon bond formation. ${ }^{1}$ It has been applied widely in the synthesis of bioactive complex molecules thanks to its mild reaction conditions and its compatibility with a broad range of functional groups such as ester, nitrile and amide. ${ }^{2}$ The recent development of multistep sequences in a single flask, such as tandem, cascade or sequential reactions is making this reaction a hot topic in organic chemistry. ${ }^{3}$ In this context and in connection with our research program on the design and synthesis of original molecules with pharmacological properties, ${ }^{4}$ the $S_{N} A r$ reaction was combined with the SuzukiMiyaura cross-coupling reaction to perform one-pot sequential polyfunctionalization of the quinazoline ring.

Quinazoline and its derivatives are important compounds widely found in natural products and synthetic pharmaceuticals. They are valued for their biological and physiological activities, such as antibacterial, ${ }^{5}$ antiviral, ${ }^{6}$ antitubercular ${ }^{7}$ and anticancer $^{8}$ activities. Several procedures for $\mathrm{S}_{\mathrm{N}} \mathrm{Ar}^{9}$ and palladium-catalyzed ${ }^{10}$ functionalization of 4chloroquinazoline have been reported, but the polyhalogenated quinazoline series has to date been little explored. ${ }^{11}$ Herein, we report an efficient procedure for the sequential polyfonctionalization of 6,8-dibromo-2,4-dichloroquinazoline 4 through a onepot chemoselective sequential bis-S $\mathrm{S}_{\mathrm{N}} \mathrm{Ar} /$ bis-SuzukiMiyaura cross-coupling reactions.

6,8-Dibromo-2,4-dichloroquinazoline 4 was obtained in four steps using 2-aminobenzonitrile as starting material in $30 \%$ overall yield as shown in Scheme 1. Bromination of 2-aminobenzonitrile with $\mathrm{N}$ bromosuccinimide (NBS) led to 2-amino-3,5dibromobenzonitrile $\mathbf{1}$ in $77 \%$ yield. Then, using an aqueous solution of $\mathrm{NaOH}$, the nitrile group was hydrolyzed into an acid group, led to compound $\mathbf{2}$ in $81 \%$ yield. Anthranilic acid 2 was converted into 6,8dibromoquinazolin-2,4-(1H,3H)-dione 3 in good yield $(76 \%)$ due to the use of urea and cesium carbonate in DMF. In the last step, the intermediate $\mathbf{3}$ was chlorinated in presence of phosphorus oxychloride and diisopropylethylamine, which leading to our key substrate 4 in $63 \%$ yield.

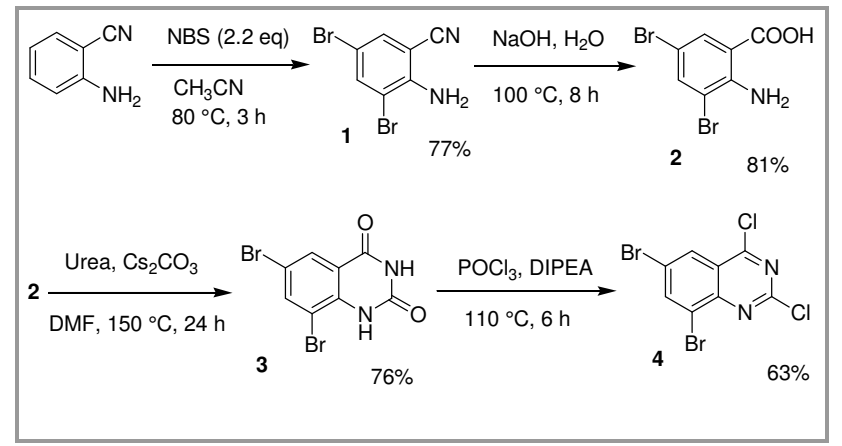

Scheme 1 Synthesis of 6,8-dibromo-2,4-dichloroquinazoline 4

When 3-chloroaniline (4 equiv) was added to 4 in $\mathrm{DME} / \mathrm{EtOH}(9 / 1)$ under microwave irradiation at 100 ${ }^{\circ} \mathrm{C}$ (Scheme 2), the corresponding chemoselective substitution product $\mathbf{5}$ was isolated in high yield (96\%) and the structure confirmed by HRMS analysis. This result indicated the high reactivity of $\mathrm{C}-2-\mathrm{Cl}$ and C-4-Cl bonds toward $\mathrm{S}_{\mathrm{N}} \mathrm{Ar}$ and no reactivity was observed with $\mathrm{C}-6-\mathrm{Br}$ and $\mathrm{C}-8-\mathrm{Br}$ bonds with a complete conversion into $\mathbf{5}$ occurred for a short time $(1 \mathrm{~h})$. However, when the reaction was performed at $80{ }^{\circ} \mathrm{C}$, a mixture of the mono and disubstituted quinazoline derivatives was observed. The $\mathrm{DME} / \mathrm{EtOH}$ mixture was chosen as solvent for the subsequent one-pot chemoselective bis- $\mathrm{S}_{\mathrm{N}} \mathrm{Ar}$ and bisSuzuki-Miyaura reactions. In previous studies, we showed the DME/EtOH mixture to be very useful for Suzuki-Miyaura cross-coupling reactions. ${ }^{12}$ 


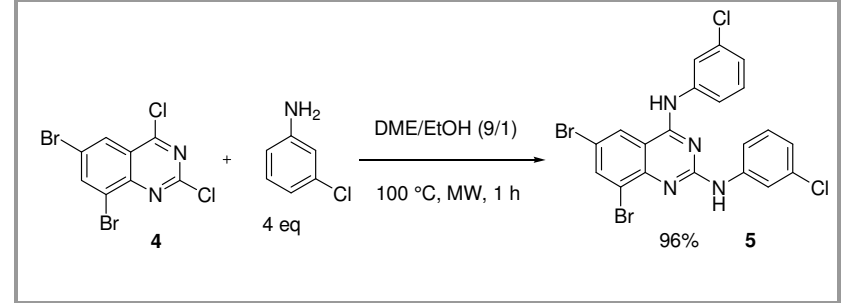

Scheme 2 Bis- $\mathrm{S}_{\mathrm{N}} \mathrm{Ar}$ chemoselective reaction of 3-chloroaniline on 4 at $\mathrm{C}-2$ and $\mathrm{C}-4$ positions

The versatility of one-pot chemoselective synthesis of 2,4,6,8-tetrasubstituted quinazoline was then assessed. One-pot multistep processes are economically advantageous in terms of the outlay for catalyst, solvent, purification materials and time. Having established the protocol to produce the bis-S $\mathrm{N} A r$ adduct at C-2 and C-4 positions of $\mathbf{4}$, we focused our attention on the one-pot chemoselective bis-S $\mathrm{S}_{\mathrm{N}} \mathrm{Ar}$ and bis-Suzuki-Miyaura reactions of 6,8-dibromo-2,4dichloroquinazoline 4.

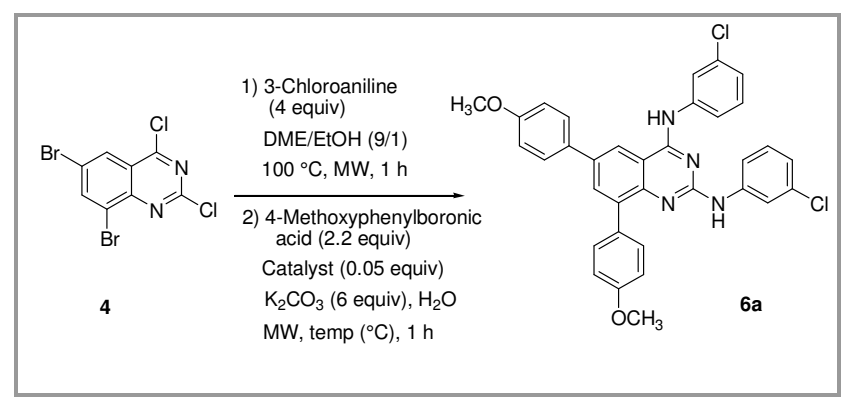

Scheme 3 One-pot chemoselective bis-S $\mathrm{N}_{\mathrm{N}} \mathrm{Ar} /$ bis-Suzuki-Miyaura reactions

The one-pot two-step bis-S $\mathrm{S}_{\mathrm{N}} \mathrm{Ar} / \mathrm{bis}-\mathrm{Suzuki}$-Miyaura reactions of compound 4 were studied using 3chloroaniline and 4-methoxyphenylboronic acid under microwave irradiation (Scheme 3). Here, we began by treating compound 4 with 3 -chloroaniline (4 equiv), expected to react selectively at the $\mathrm{C}-2,4$ positions, in a mixture of $\mathrm{DME} / \mathrm{EtOH}(9 / 1)$ for $1 \mathrm{~h}$ at $100{ }^{\circ} \mathrm{C}$ under MW irradiation. After cooling to room temperature, 4methoxyphenylboronic acid (2.2 equiv), $\mathrm{PdCl}_{2}\left(\mathrm{PPh}_{3}\right)_{2}$ ( 0.05 equiv), $\mathrm{K}_{2} \mathrm{CO}_{3}$ (6 equiv) and $\mathrm{H}_{2} \mathrm{O}(0.5 \mathrm{~mL})$ were added and the reaction mixture was irradiated again for $1 \mathrm{~h}$ at $110{ }^{\circ} \mathrm{C}$. These sequences afforded the desired polysubstituted quinazoline $\mathbf{6 a}$ in an overall yield of $84 \%$ (entry 1 , Table 1 ). In addition to compound $\mathbf{6 a}$, we observed by LC-MS analysis the presence of the product of a palladium-catalyzed reduction of aromatic halides, as previously observed in the literature. ${ }^{13}$ When the reaction was performed with $\mathrm{Pd}\left(\mathrm{PPh}_{3}\right)_{4}$ as catalyst, compound 6a was obtained in somewhat lower yield $(71 \%$, entry 2$)$. However, the desired compound 6a was isolated in good yield when the temperature of the second step was decreased to $100{ }^{\circ} \mathrm{C}$ (entry 3, 90\%). In these conditions, only traces of reducted product were observed. The use of DMF as solvent instead of DME, gave a low yield of compound $\mathbf{6 a}$ (79\%, entry 5, Table 1 ) and the complete diamination reaction of the first step took longer to achieve $(2 \mathrm{~h})$.

\begin{tabular}{|c|c|c|c|c|}
\hline Entry & Catalyst & Solvent & $\begin{array}{c}\text { Temp } \\
\text { (step 2) }\end{array}$ & Yield $6 \mathbf{a}$ \\
\hline 1 & $\mathrm{PdCl}_{2}\left(\mathrm{PPh}_{3}\right)_{2}(5 \%)$ & $\mathrm{DME} / \mathrm{EtOH}$ & $110^{\circ} \mathrm{C}$ & $84 \%$ \\
\hline 2 & $\mathrm{Pd}\left(\mathrm{PPh}_{3}\right)_{4} \quad$ (5\%) & $\mathrm{DME} / \mathrm{EtOH}$ & $110^{\circ} \mathrm{C}$ & $71 \%$ \\
\hline 3 & $\mathrm{PdCl}_{2}\left(\mathrm{PPh}_{3}\right)_{2}(5 \%)$ & $\mathrm{DME} / \mathrm{EtOH}$ & $100{ }^{\circ} \mathrm{C}$ & $90 \%$ \\
\hline 4 & $\mathrm{Pd}\left(\mathrm{PPh}_{3}\right)_{4} \quad(5 \%)$ & $\mathrm{DME} / \mathrm{EtOH}$ & $100^{\circ} \mathrm{C}$ & $76 \%$ \\
\hline 5 & $\mathrm{PdCl}_{2}\left(\mathrm{PPh}_{3}\right)_{2}(5 \%)$ & $\mathrm{DMF} / \mathrm{EtOH}$ & $100^{\circ} \mathrm{C}$ & $79 \%$ \\
\hline
\end{tabular}

Once the optimized conditions were established, the scope of this one-pot bis-S $\mathrm{S}_{\mathrm{N}} \mathrm{Ar} /$ bis-Suzuki-Miyaura sequence was explored by using 3-chloroaniline for the first step and a wide variety of aryl or heteroarylboronic acids for the second step (Table 2).

Table 2 One-pot chemoselective reaction of 4 with 3-chloroaniline and various boronic acids
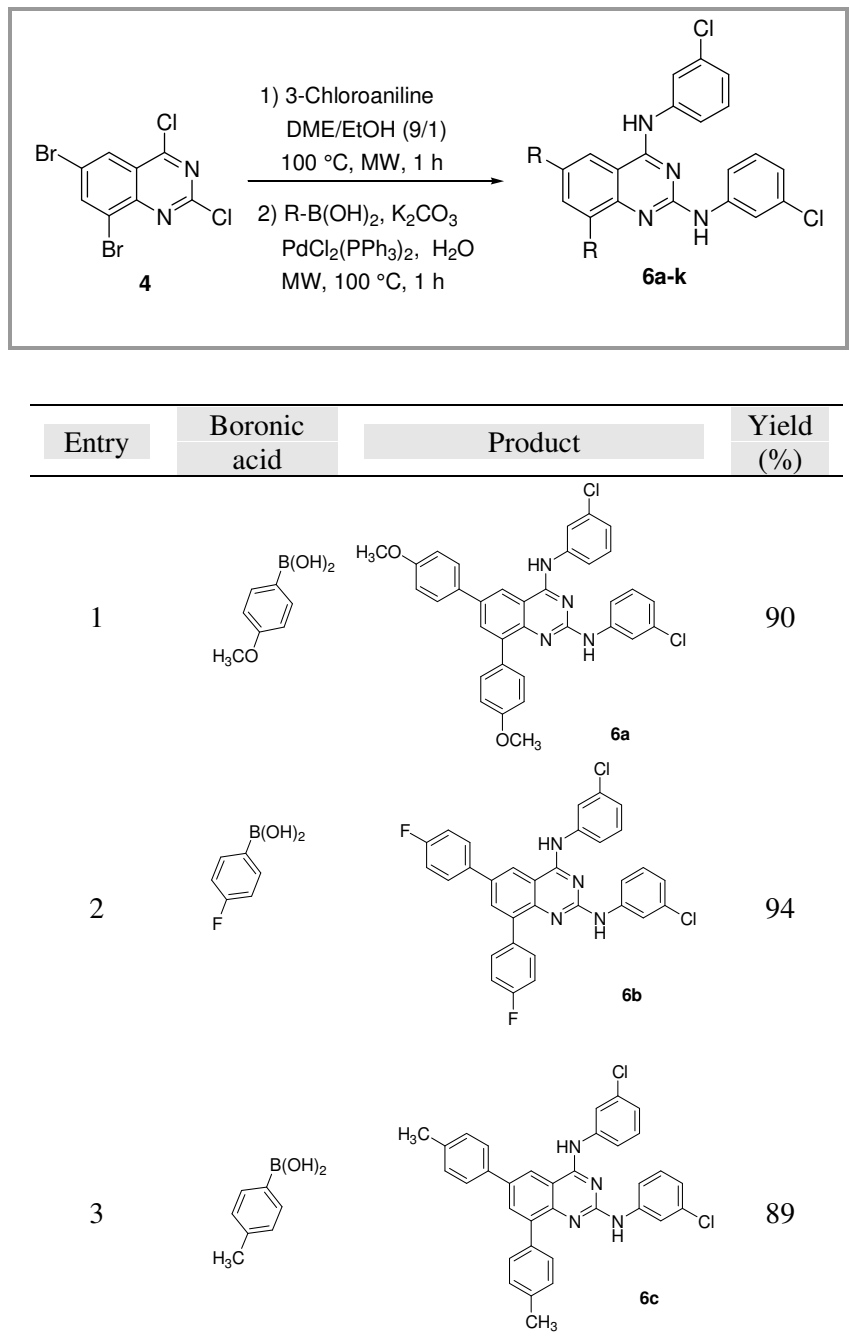


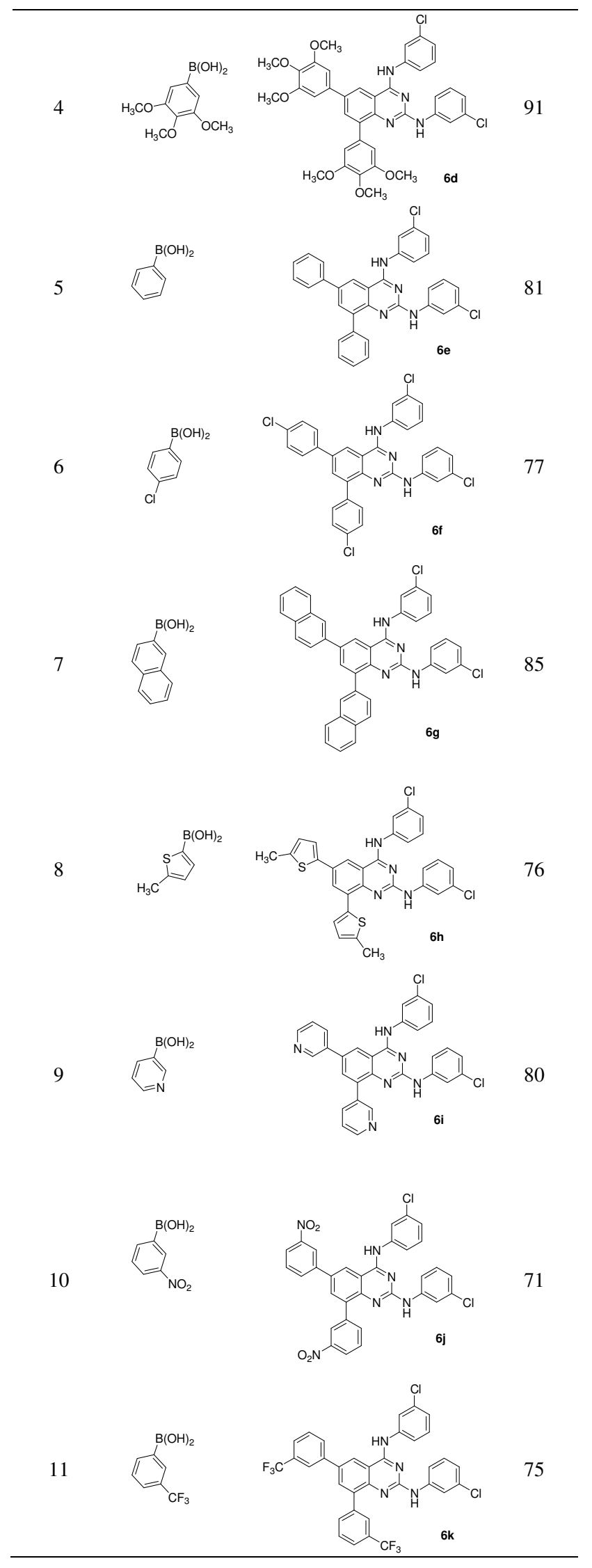

Reaction conditions: Step 1; 3-chloroaniline (4 equiv), DME/EtOH (9/1), $\mathrm{MW}, 100{ }^{\circ} \mathrm{C}, 1 \mathrm{~h}$. Step 2; boronic acids (2.2 equiv), $\mathrm{PdCl}_{2}\left(\mathrm{PPh}_{3}\right)_{2}$ (0.05 equiv), $\mathrm{K}_{2} \mathrm{CO}_{3}$ (6 equiv), $\mathrm{H}_{2} \mathrm{O}, \mathrm{MW}, 100{ }^{\circ} \mathrm{C}, 1 \mathrm{~h}$.
Good to excellent yields of tetrasubstituted quinazolines $\mathbf{6 a - k}$ were obtained with a wide range of arylboronic acids bearing electron-donating, electronwithdrawing and sterically bulky groups (entries 1-7, 10, 11, Table 2). The same good results were observed with heteroarylboronic acids (entries 8-9, Table 2).

Under the same microwave conditions, the scope of the one-pot reaction was investigated using various amines for the first step and 4-fluorophenylboronic acid for the second step (Table 3). Various aromatic and aliphatic amines were used for the preparation of a new series of compound $\mathbf{6}$ derivatives, including anilines, primary and secondary amines. The use of aniline derivatives with no steric hindrance gave in good to excellent yields of the corresponding products under the optimal conditions (Table 3, entries 1, 2 and 5). However, slightly low reactivity was observed with aniline derivatives with steric hindrance toward the bis- $\mathrm{S}_{\mathrm{N}} \mathrm{Ar}$ under these experimental conditions. By increasing the reaction temperature of the first step from $100{ }^{\circ} \mathrm{C}$ (Table 3, entry 3, 4 and 6) to $110{ }^{\circ} \mathrm{C}$ and finally to $130{ }^{\circ} \mathrm{C}$ and increasing the reaction time to 2 $\mathrm{h}$, complete conversion (bis-S $\mathrm{S}_{\mathrm{N}} \mathrm{Ar} /$ bis-Suzuki-Miyaura cross-coupling) of the compound $\mathbf{4}$ was achieved (as monitored by LC-MS) affording the desired products in good yields. With primary and secondary amines, the complete conversion of compound 4 into the bis$\mathrm{S}_{\mathrm{N}} \mathrm{Ar}$ adduct during the first step was achieved after 2 $\mathrm{h}$ at $130{ }^{\circ} \mathrm{C}$. Then, after $1 \mathrm{~h}$ under the cross-coupling reaction conditions, the corresponding products $6 \mathbf{r}-\mathbf{t}$ were isolated in very good yields (entries 7-9, Table 3).

Table 3 One-pot chemoselective reaction of $\mathbf{4}$ with various amines and 4-fluorophenylboronic acid
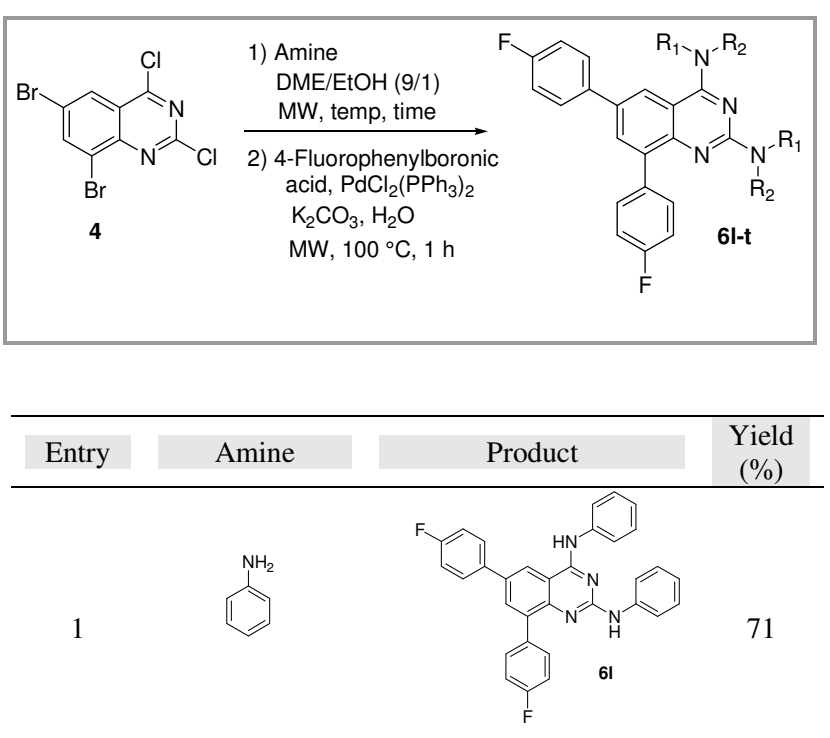


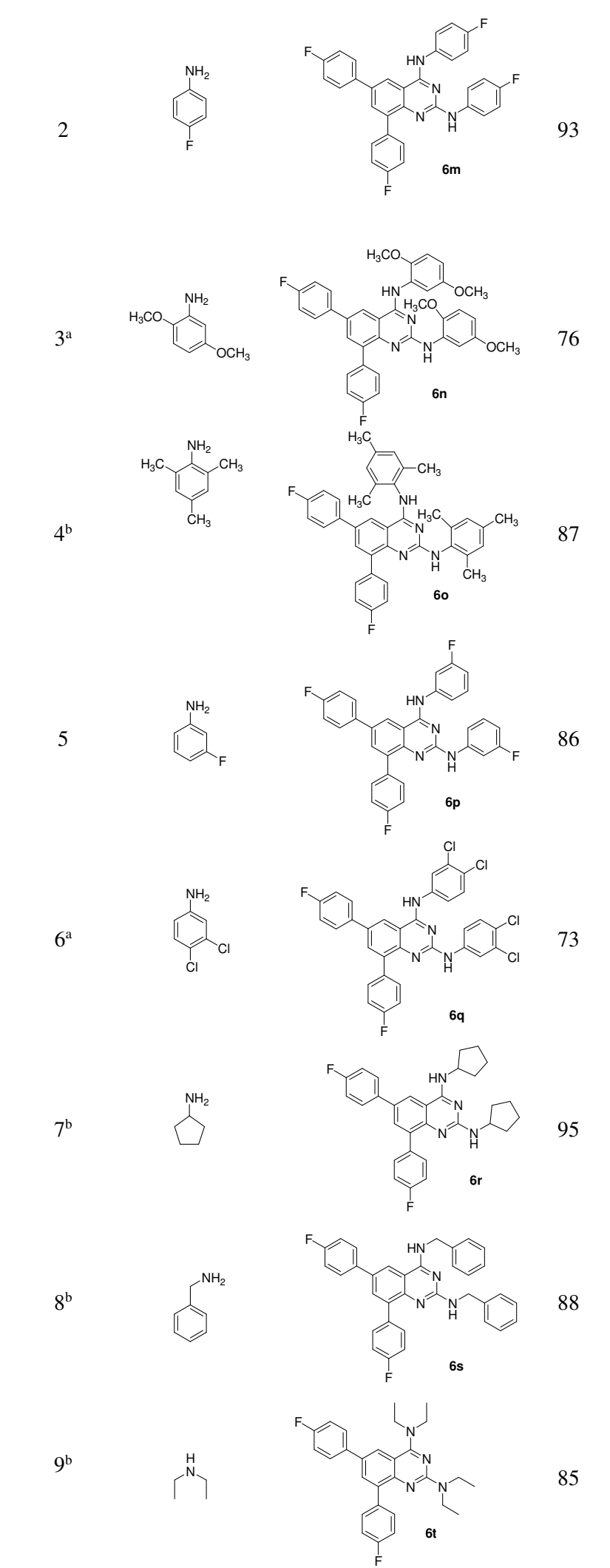

Reaction conditions: Step 1; amine (4 equiv), DME/EtOH (9/1), MW, $100{ }^{\circ} \mathrm{C}, 1 \mathrm{~h}$. Step 2; 4-fluorophenylboronic acid (2.2 equiv), $\mathrm{PdCl}_{2}\left(\mathrm{PPh}_{3}\right)_{2}$ (0.05 equiv), $\mathrm{K}_{2} \mathrm{CO}_{3}$ (6 equiv), $\mathrm{H}_{2} \mathrm{O}, \mathrm{MW}, 100{ }^{\circ} \mathrm{C}, 1$ h. a Step 1 ; MW, $110^{\circ} \mathrm{C}, 2 \mathrm{~h}$. ${ }^{\mathrm{b}}$ Step $1 ; \mathrm{MW}, 130^{\circ} \mathrm{C}, 2 \mathrm{~h}$.

In summary, we report here the synthesis of 6,8dibromo-2,4-dichloroquinazoline $\mathbf{4}$ and its controlled chemoselectivity diversification through various combinations of $\mathrm{S}_{\mathrm{N}} \mathrm{Ar}$ and cross-coupling SuzukiMiyaura reactions. This approach allows rapid and efficient access to 2,4,6,8-tetrasubstituted quinazoline derivatives with high yields using a one-pot chemoselective methodology via consecutive bis$\mathrm{S}_{\mathrm{N}} \mathrm{Ar} /$ bis-Suzuki-Miyaura reactions under microwave irradiation. In addition, this strategy tolerates a wide range of boronic acids and amines and could rapidly produce numerous and varied analogues of polysubstituted quinazoline derivatives potentially bioactive heterocyclic compounds.

Melting points were determined on Büchi B-540 and are uncorrected. Elemental analyses and HRMS were carried out at the Spectropole, Faculté des Sciences et Techniques de Saint-Jérôme, Marseille. $200 \mathrm{MHz}{ }^{1} \mathrm{H}$ NMR (reference $\mathrm{CDCl}_{3} \delta=7.26$, DMSO- $d_{6} \delta=2.50$ ) and $50 \mathrm{MHz}{ }^{13} \mathrm{C}$ NMR spectra (reference $\mathrm{CDCl}_{3} \delta=$ 77.0, DMSO- $d_{6} \delta=39.7$ ) were recorded on a Bruker ARX 200 spectometer in $\mathrm{CDCl}_{3}$ and DMSO- $d_{6}$ solvents at the Faculté de Pharmacie de Marseille. Solvents were dried by conventional methods. The following adsorbent was used for column chromatography: silica gel 60 (Merck, particle size 0.063-0.200 mm, 70-230 mesh ASTM). TLC was performed on $5 \mathrm{~cm} \times 10 \mathrm{~cm}$ aluminium plates coated with silica gel 60F-254 (Merck) in an appropriate eluent. HRMS spectra were recorded on QStar Elite (Applied Biosystems SCIEX) spectrometter. PEG was the matrix for HRMS.

Microwave reactions were performed with a Biotage ${ }^{\circledR}$ Initiator Microwave oven using 2-5 mL sealed vials; temperatures were measured with an IR-sensor and reaction times are given as hold times.

LC/MS analyses were performed at the Faculté de Pharmacie de Marseille on an Accela High System ${ }^{\circledR}$ chain U-HPLC coupled with a Thermo MSQ Plus ${ }^{\circledR}$ simple quadripole. A Thermo Hypersil Gold ${ }^{\circledR} 50 \times 2.1$ $\mathrm{mm}$ chromatographic column was used $\left(\mathrm{SiO}_{2} \mathrm{C18}\right)$ with $1.9 \mu \mathrm{m}$ diameter particles. The analysis is $8 \mathrm{~min}$ running, with a $\mathrm{MeOH} / \mathrm{H}_{2} \mathrm{O}$ gradient eluent from $50 / 50$ to $95 / 05$.

\section{Acknowledgments}

This work was supported by the CNRS (Centre National de la Recherche Scientifique) and Aix-Marseille Université. The authors thank V. Remusat for NMR spectra recording and the Spectropole team for various analytical measurements.

\section{2-Amino-3,5-dibromobenzonitrile (1)}

A mixture of 2-aminobenzonitrile ( $5 \mathrm{~g}, 42.3 \mathrm{mmol}$ ) and NBS (16.57 g, $93.1 \mathrm{mmol}, 2.2$ equiv) was refluxed in $\mathrm{MeCN}(80 \mathrm{~mL}$ ) for $3 \mathrm{~h}$. After cooling, a precipitate appeared and was filtered, washed with water $(3 \times 60 \mathrm{~mL})$ and dried in a vacuum drying oven (desiccator cabinet). Recrystallization from propan-2ol gave 1 as brown solid; yield 77\% (8.9 g); mp 158 ${ }^{\circ} \mathrm{C}$ (Lit. $\left.{ }^{14} 156{ }^{\circ} \mathrm{C}\right)$. 
${ }^{1} \mathrm{H} \mathrm{NMR}\left(200 \mathrm{MHz}, \mathrm{CDCl}_{3}\right): \delta=4.91\left(\mathrm{~s}, 2 \mathrm{H}, \mathrm{NH}_{2}\right)$, $7.47(\mathrm{~d}, J=2.1 \mathrm{~Hz}, 1 \mathrm{H}, \mathrm{H}-4), 7.71(\mathrm{~d}, J=2.1 \mathrm{~Hz}, 1$ $\mathrm{H}, \mathrm{H}-6)$.

${ }^{13} \mathrm{C} \mathrm{NMR}\left(50 \mathrm{MHz}, \mathrm{CDCl}_{3}\right): \delta=97.9,108.2,109.7$, 115.6, 133.6, 139.4, 146.2.

\section{2-Amino-3,5-dibromobenzoic acid (2)}

To a solution of 2-amino-3,5-dibromobenzonitrile 1 (8 $\mathrm{g}, 29.0 \mathrm{mmol})$ in water $(100 \mathrm{~mL}), \mathrm{NaOH}(1.16 \mathrm{~g}, 1$ equiv) was added. The reaction mixture was heated at $100{ }^{\circ} \mathrm{C}$ for $8 \mathrm{~h}$. After cooling, a precipitate appeared and was filtered, washed with water (3 x $60 \mathrm{~mL})$ and dried in a vacuum drying oven (desiccator cabinet). Recrystallization from propan-2-ol gave $\mathbf{2}$ as yellow solid; yield 81\% (6.58 g); mp $223{ }^{\circ} \mathrm{C}$ (Lit. ${ }^{15} 226-228$ $\left.{ }^{\circ} \mathrm{C}\right)$.

${ }^{1} \mathrm{H}$ NMR (200 MHz, DMSO-d $\left.)_{6}\right): \delta=6.80(\mathrm{~s}, 2 \mathrm{H}$, $\mathrm{NH}_{2}$ ), 7.72 (d, $\left.J=1.9 \mathrm{~Hz}, 1 \mathrm{H}, \mathrm{H}-4\right), 7.78$ (d, $J=1.9$ $\mathrm{Hz}, 1 \mathrm{H}, \mathrm{H}-6), 8.05$ (s, $1 \mathrm{H}, \mathrm{COOH})$.

${ }^{13} \mathrm{C}$ NMR (50 MHz, DMSO-d $)$ : $\delta=105.1,110.6$, 117.0, 130.9, 136.8, 146.2, 169.4.

\section{6,8-Dibromoquinazoline-2,4-(1H,3H)-dione (3)}

A mixture of 2-amino-3,5-dibromobenzoic acid 2 (5 $\mathrm{g}, 16.95 \mathrm{mmol})$, urea $(5.09 \mathrm{~g}, 84.75 \mathrm{mmol}, 5$ equiv) and $\mathrm{Cs}_{2} \mathrm{CO}_{3}(0.55 \mathrm{~g}, 1.69 \mathrm{mmol}, 0.1$ equiv) was refluxed in DMF (80 mL) for $24 \mathrm{~h}$. After cooling, a precipitate appeared and was filtered, washed with water $(3 \times 60 \mathrm{~mL})$ and dried in a vacuum drying oven (desiccator cabinet). Recrystallization from propan-2ol gave 3 as brown solid; yield 76\% (4.14 g); mp 289 ${ }^{\circ} \mathrm{C}$ (Lit. ${ }^{16} 291-292{ }^{\circ} \mathrm{C}$ ).

${ }^{1} \mathrm{H}$ NMR $\left(200 \mathrm{MHz}, \mathrm{DMSO}-d_{6}\right): \delta=7.84(\mathrm{~d}, J=2.2$ Hz, $1 \mathrm{H}, \mathrm{H}-6), 7.94$ (d, $J=2.2 \mathrm{~Hz}, 1 \mathrm{H}, \mathrm{H}-8), 10.76$ (s, $2 \mathrm{H}, 2 \mathrm{NH})$.

${ }^{13} \mathrm{C}$ NMR (50 MHz, DMSO-d 6 ): $\delta=110.4,114.0$, 118.0, 128.5, 138.8, 145.1, 153.4, 162.7.

\section{6,8-Dibromo-2,4-dichloroquinazoline (4)}

A solution of 6,8-dibromoquinazoline-2,4-(1H,3H)dione 3 ( $4 \mathrm{~g}, 12.5 \mathrm{mmol}), \mathrm{POCl}_{3}$ (11.4 mL, 10 equiv), diisopropyl ethylamine ( $4.4 \mathrm{~mL}, 25.0 \mathrm{mmol}, 2$ equiv) was heated at reflux for $6 \mathrm{~h}$. After this time, the reaction mixture was poured with care over crushed ice and stirred vigorously. This aqueous mixture was extracted with $\mathrm{CH}_{2} \mathrm{Cl}_{2}$, and the combined organic layers were washed with brine, dried over $\mathrm{Na}_{2} \mathrm{SO}_{4}$ and evaporated under reduced pressure. Flash chromatography (silica gel, $\mathrm{CH}_{2} \mathrm{Cl}_{2}$ /petroleum ether, $2 / 1$ ) provided after recrystallization from propan-2-ol 4 as yellow solid; yield 63\% (2.81 g); mp $145{ }^{\circ} \mathrm{C}$ (Lit. ${ }^{17} 148-149^{\circ} \mathrm{C}$ ).

${ }^{1} \mathrm{H}$ NMR $\left(200 \mathrm{MHz}, \mathrm{CDCl}_{3}\right): \delta=8.37(\mathrm{~d}, J=2.0 \mathrm{~Hz}$, $1 \mathrm{H}, \mathrm{H}-6), 8.39$ (d, $J=2.0 \mathrm{~Hz}, 1 \mathrm{H}, \mathrm{H}-8)$.

${ }^{13} \mathrm{C}$ NMR (50 MHz, $\left.\mathrm{CDCl}_{3}\right): \delta=122.7,124.1,124.2$, 127.7, 142.3, 149.0, 156.3, 163.3.

\section{6,8-Dibromo- $N^{2}, N^{4}$-bis(3-chlorophenyl)-} quinazoline-2,4-diamine (5)
A solution of 6,8-dibromo-2,4-dichloroquinazoline 4 (0.2 g, $0.56 \mathrm{mmol})$, 3-chloroaniline $(0.24 \mathrm{~mL}, 2.24$ mmol, 4 equiv) in a DME/EtOH mixture $(9 / 1,5 \mathrm{~mL})$ was heated at $100{ }^{\circ} \mathrm{C}$ under microwave irradiation for $1 \mathrm{~h}$. After cooling, a precipitate appeared and was filtered, washed with water $(3 \times 10 \mathrm{~mL})$ and dried in a vacuum drying oven (desiccator cabinet). Recrystallization from propan-2-ol gave $\mathbf{5}$ as yellow solid; yield 96\% (0.29 g); $\mathrm{mp} 222{ }^{\circ} \mathrm{C}$.

${ }^{1} \mathrm{H}$ NMR (200 MHz, DMSO- $\left.d_{6}\right): \delta=7.01(\mathrm{~d}, J=6.3$ $\mathrm{Hz}, 1 \mathrm{H}), 7.19-7.34(\mathrm{~m}, 2 \mathrm{H}), 7.43(\mathrm{t}, J=7.7 \mathrm{~Hz}, 1 \mathrm{H})$, $7.81(\mathrm{~d}, J=8.2 \mathrm{~Hz}, 1 \mathrm{H}), 7.93(\mathrm{~s}, 1 \mathrm{H}), 8.02(\mathrm{~d}, J=$ $8.2 \mathrm{~Hz}, 1 \mathrm{H}), 8.23$ (s, $1 \mathrm{H}), 8.48$ (s, $1 \mathrm{H}), 8.75$ (s, $1 \mathrm{H})$, 9.81 (s, $1 \mathrm{H}, \mathrm{NH}), 9.93$ (s, $1 \mathrm{H}, \mathrm{NH})$.

${ }^{13} \mathrm{C}$ NMR (50 MHz, DMSO-d $)$ : $\delta=114.1,114.4$, $117.7,118.9,121.0,121.3,121.9,124.0,125.6,130.1$, $130.4,133.1,133.3,138.5,140.5,142.0,147.6,156.1$, 157.7 .

HRMS (ESI): $\mathrm{m} / \mathrm{z}[\mathrm{M}+\mathrm{H}]^{+}$Calcd for $\mathrm{C}_{20} \mathrm{H}_{12} \mathrm{Br}_{2} \mathrm{Cl}_{2} \mathrm{~N}_{4}$ : 538.8857; found: 538.8858 .

General procedure of the one-pot chemoselective bis-S $\mathrm{S}_{\mathrm{N}} \mathrm{Ar}$ and bis-Suzuki-Miyaura reactions

\section{Synthesis of compounds 6a-k}

A solution of 6,8-dibromo-2,4-dichloroquinazoline 4 (0.1 g, $0.28 \mathrm{mmol})$, 3-chloroaniline $(0.12 \mathrm{~mL}, 1.12$ mmol, 4 equiv) in a DME/EtOH mixture $(9 / 1,5 \mathrm{~mL})$ was heated at $100{ }^{\circ} \mathrm{C}$ under microwave irradiation for $1 \mathrm{~h}$. After cooling, boronic acid (0.62 mmol, 2.2 equiv), $\mathrm{PdCl}_{2}\left(\mathrm{PPh}_{3}\right)_{2}$ (10 mg, $14 \mu \mathrm{mol}, 0.05$ equiv), $\mathrm{K}_{2} \mathrm{CO}_{3}(0.23 \mathrm{~g}, 1.68 \mathrm{mmol}, 6$ equiv) and water $(0.5$ $\mathrm{mL})$ were introduced under argon. The mixture was heated at $100{ }^{\circ} \mathrm{C}$ for $1 \mathrm{~h}$ under microwave irradiation. After cooling, $80 \mathrm{~mL}$ of water were added and the solution was extracted with dichloromethane $(3 \times 60$ $\mathrm{mL})$. The organic layer was washed with water $(3 \mathrm{x}$ $100 \mathrm{~mL}$ ), dried over $\mathrm{Na}_{2} \mathrm{SO}_{4}$ and evaporated. The crude product was purified by column chromatography (silica gel, petroleum ether/ethyl acetate $(9 / 1)$, (8/2 for $\mathbf{6 d})$, ethyl acetate for $\mathbf{6 i})$ ) and recrystallized from acetonitrile.

$N^{2}, N^{4}$-Bis(3-chlorophenyl)-6,8-bis(4-methoxyphenyl)quinazoline-2,4-diamine (6a)

Yellow powder; yield 90\% (0.15 g); mp $156{ }^{\circ} \mathrm{C}$ ${ }^{1} \mathrm{H}$ NMR (200 MHz, DMSO- $\left.d_{6}\right): \delta=3.83$ (s, $6 \mathrm{H}, 2$ $\left.\mathrm{OCH}_{3}\right), 6.89-7.31(\mathrm{~m}, 7 \mathrm{H}), 7.45(\mathrm{~s}, 1 \mathrm{H}), 7.70-8.08$ $(\mathrm{m}, 9 \mathrm{H}), 8.61$ (s, $1 \mathrm{H}), 9.38$ (s, $1 \mathrm{H}, \mathrm{NH}), 9.83$ (s, 1 $\mathrm{H}, \mathrm{NH})$.

${ }^{13} \mathrm{C}$ NMR (50 MHz, DMSO- $\left.d_{6}\right): \delta=55.3\left(\mathrm{OCH}_{3}\right)$, $55.4\left(\mathrm{OCH}_{3}\right), 112.9,113.7,114.5,117.2,117.8,118.9$, $120.5,120.7,121.6,123.3,128.2,129.8,130.4,131.4$, $131.7,132.0,132.1,133.1,133.2,134.0,137.5,141.3$, $142.6,148.3,155.2,158.7,159.1$.

HRMS (ESI): $\mathrm{m} / \mathrm{z}[\mathrm{M}+\mathrm{H}]^{+}$Calcd for $\mathrm{C}_{34} \mathrm{H}_{26} \mathrm{Cl}_{2} \mathrm{~N}_{4} \mathrm{O}_{2}$ : 593.1506; found: 593.1506 .

$N^{2}, N^{4}$-Bis(3-chlorophenyl)-6,8-bis(4-fluorophenyl)quinazoline-2,4-diamine (6b) 
Yellow powder; yield $94 \%(0.15 \mathrm{~g}) ; \mathrm{mp} 178{ }^{\circ} \mathrm{C}$

${ }^{1} \mathrm{H}$ NMR $\left(200 \mathrm{MHz}\right.$, DMSO- $\left.d_{6}\right): \delta=6.89(\mathrm{~d}, J=8.4$ $\mathrm{Hz}, 1 \mathrm{H}), 7.12-7.22$ (m, $2 \mathrm{H}), 7.28-7.49$ (m, $5 \mathrm{H}), 7.62$ $(\mathrm{d}, J=8.4 \mathrm{~Hz}, 1 \mathrm{H}), 7.74-7.81(\mathrm{~m}, 2 \mathrm{H}), 7.91-7.98(\mathrm{~m}$, $4 \mathrm{H}), 8.06-8.13(\mathrm{~m}, 2 \mathrm{H}), 8.68(\mathrm{~s}, 1 \mathrm{H}), 9.44(\mathrm{~s}, 1 \mathrm{H}$, $\mathrm{NH}), 9.86$ (s, $1 \mathrm{H}, \mathrm{NH})$.

${ }^{13} \mathrm{C}$ NMR (50 MHz, DMSO- $\left.d_{6}\right): \delta=112.8,115.0(\mathrm{~d}, J$ $=21.2 \mathrm{~Hz}), 115.9(\mathrm{~d}, J=21.6 \mathrm{~Hz}), 117.3,117.9$, $120.3,120.7,120.8,121.7,123.4,129.1(\mathrm{~d}, J=8.1$ $\mathrm{Hz}), 129.8,130.4,132.2(\mathrm{~d}, J=8.1 \mathrm{~Hz}), 132.4,133.1$, $133.2,135.6(\mathrm{~d}, J=2.9 \mathrm{~Hz}), 136.1(\mathrm{~d}, J=2.9 \mathrm{~Hz})$, $136.9,141.1,142.5,148.7,155.5,158.7,161.8$ (d, $J=$ $243.7 \mathrm{~Hz}), 162.1(\mathrm{~d}, J=244.4 \mathrm{~Hz})$.

Anal. Calcd for $\mathrm{C}_{32} \mathrm{H}_{20} \mathrm{Cl}_{2} \mathrm{~F}_{2} \mathrm{~N}_{4}: \mathrm{C}, 67.50 ; \mathrm{H}, 3.54 ; \mathrm{N}$, 9.84. Found: C, 67.56; H, 3.21; N, 10.23.

$N^{2}, N^{4}$-Bis(3-chlorophenyl)-6,8-dip-tolylquinazoline-2,4-diamine (6c)

Yellow powder; yield $89 \%(0.14 \mathrm{~g})$; mp $177{ }^{\circ} \mathrm{C}$

${ }^{1} \mathrm{H}$ NMR $\left(200 \mathrm{MHz}\right.$, DMSO- $\left.d_{6}\right): \delta=2.37(\mathrm{~s}, 3 \mathrm{H}$, $\left.\mathrm{CH}_{3}\right), 2.40\left(\mathrm{~s}, 3 \mathrm{H}, \mathrm{CH}_{3}\right), 6.88(\mathrm{~d}, J=7.9 \mathrm{~Hz}, 1 \mathrm{H})$, 7.10-7.22 (m, $2 \mathrm{H}), 7.33(\mathrm{~d}, J=7.5 \mathrm{~Hz}, 4 \mathrm{H}), 7.45(\mathrm{t}$, $J=8.2 \mathrm{~Hz}, 1 \mathrm{H}), 7.63(\mathrm{~d}, J=7.8 \mathrm{~Hz}, 2 \mathrm{H}), 7.70(\mathrm{~s}, 1$ $\mathrm{H}), 7.80(\mathrm{~d}, J=7.8 \mathrm{~Hz}, 2 \mathrm{H}), 7.95-7.98(\mathrm{~m}, 2 \mathrm{H})$, 8.07-8.14 (m, $2 \mathrm{H}), 8.67$ (s, $1 \mathrm{H}), 9.40(\mathrm{~s}, 1 \mathrm{H}, \mathrm{NH})$, $9.85(\mathrm{~s}, 1 \mathrm{H}, \mathrm{NH})$.

${ }^{13} \mathrm{C}$ NMR (50 MHz, DMSO- $\left.d_{6}\right): \delta=20.9\left(\mathrm{CH}_{3}\right), 21.1$ $\left(\mathrm{CH}_{3}\right), 112.8,117.2,117.8,119.5,120.6,120.8,121.7$, $123.3,126.9,128.9,129.7,130.1,130.4,132.1,133.1$, $133.2,134.2,136.3,136.5,136.8,136.9,137.9,141.2$, 142.6, 148.6, 155.3, 158.7 .

Anal. Calcd for $\mathrm{C}_{34} \mathrm{H}_{26} \mathrm{Cl}_{2} \mathrm{~N}_{4}$ : C, 72.73; H, 4.67; N, 9.98. Found: C, 72.34; H, 4.64; N, 9.82.

$N^{2}, N^{4}$-Bis(3-chlorophenyl)-6,8-bis $(3,4,5-$ trimethoxyphenyl)quinazoline-2,4-diamine (6d)

Yellow powder; yield $91 \%(0.18 \mathrm{~g}) ; \mathrm{mp} 269^{\circ} \mathrm{C}$

${ }^{1} \mathrm{H}$ NMR $\left(200 \mathrm{MHz}\right.$, DMSO- $\left.d_{6}\right): \delta=3.73(\mathrm{~s}, 3 \mathrm{H}$, $\left.\mathrm{OCH}_{3}\right), 3.76\left(\mathrm{~s}, 3 \mathrm{H}, \mathrm{OCH}_{3}\right), 3.79\left(\mathrm{~s}, 6 \mathrm{H}, 2 \mathrm{OCH}_{3}\right)$, $3.93\left(\mathrm{~s}, 6 \mathrm{H}, 2 \mathrm{OCH}_{3}\right), 6.89(\mathrm{~d}, J=2.6 \mathrm{~Hz}, 1 \mathrm{H}), 7.01$ $(\mathrm{s}, 2 \mathrm{H}), 7.11(\mathrm{~s}, 2 \mathrm{H}), 7.16-7.22(\mathrm{~m}, 2 \mathrm{H}), 7.47(\mathrm{t}, J=$ $8.0 \mathrm{~Hz}, 1 \mathrm{H}), 7.82(\mathrm{~d}, J=7.6 \mathrm{~Hz}, 1 \mathrm{H}), 8.03(\mathrm{~d}, J=$ $6.8 \mathrm{~Hz}, 2 \mathrm{H}), 8.11-8.15(\mathrm{~m}, 2 \mathrm{H}), 8.62(\mathrm{~s}, 1 \mathrm{H}), 9.47$ (s, $1 \mathrm{H}, \mathrm{NH}), 9.82(\mathrm{~s}, 1 \mathrm{H}, \mathrm{NH})$.

${ }^{13} \mathrm{C}$ NMR $\left(50 \mathrm{MHz}, \mathrm{DMSO}-d_{6}\right): \delta=55.8\left(2 \mathrm{OCH}_{3}\right)$, $56.4\left(2 \mathrm{OCH}_{3}\right), 60.1\left(\mathrm{OCH}_{3}\right), 60.4\left(\mathrm{OCH}_{3}\right), 105.1$, 107.8, 112.7, 117.0, 117.8, 120.4, 120.5, 120.8, 121.7, $123.4,129.7,130.5,132.9,133.1,133.3,134.7,135.1$, $135.8,136.9,137.5,138.0,141.2,142.6,148.6,152.6$, $153.5,155.4,158.7$.

Anal. Calcd for $\mathrm{C}_{38} \mathrm{H}_{34} \mathrm{Cl}_{2} \mathrm{~N}_{4} \mathrm{O}_{6}: \mathrm{C}, 63.96 ; \mathrm{H}, 4.80 ; \mathrm{N}$, 7.85. Found: C, 63.83; H, 4.82; N, 7.74.

$N^{2}, N^{4}$-Bis(3-chlorophenyl)-6,8-diphenylquinazoline-2,4-diamine (6e)

Yellow powder; yield $81 \%(0.12 \mathrm{~g})$; mp $168{ }^{\circ} \mathrm{C}$ ${ }^{1} \mathrm{H}$ NMR $\left(200 \mathrm{MHz}, \mathrm{DMSO}-d_{6}\right): \delta=6.88(\mathrm{~d}, J=7.0$ $\mathrm{Hz}, 1 \mathrm{H}), 7.11-7.22$ (m, 2 H), 7.46-7.53 (m, 7 H), 7.74 (bs, $3 \mathrm{H}), 7.90-8.14$ (m, $6 \mathrm{H}), 8.73$ (s, $1 \mathrm{H}), 9.43$ (s, 1 $\mathrm{H}, \mathrm{NH}), 9.89$ (s, $1 \mathrm{H}, \mathrm{NH})$.

${ }^{13} \mathrm{C}$ NMR $\left(50 \mathrm{MHz}, \mathrm{DMSO}-d_{6}\right): \delta=112.9,117.3$, $117.9,120.3,120.6,120.8,121.7,123.4,127.1,127.2$, $127.6,128.2,129.1,129.8,130.4,132.6,133.1,134.3$, $137.9,139.5,139.7,141.2,142.5,148.8,155.5,158.8$.

Anal. Calcd for $\mathrm{C}_{32} \mathrm{H}_{22} \mathrm{Cl}_{2} \mathrm{~N}_{4}$ : C, 72.05; H, 4.16; N, 10.50. Found: C, 71.92; H, 4.13; N, 10.42 .

$N^{2}, N^{4}$-Bis(3-chlorophenyl)-6,8-bis(4-chlorophenyl)quinazoline-2,4-diamine (6f)

Yellow powder; yield $77 \%(0.13 \mathrm{~g}) ; \mathrm{mp} 261{ }^{\circ} \mathrm{C}$

${ }^{1} \mathrm{H}$ NMR $\left(200 \mathrm{MHz}\right.$, DMSO- $\left.d_{6}\right): \delta=6.90(\mathrm{~d}, J=7.0$ $\mathrm{Hz}, 1 \mathrm{H}), 7.11-7.22(\mathrm{~m}, 2 \mathrm{H}), 7.43(\mathrm{~d}, J=8.0 \mathrm{~Hz}, 1$ H), 7.50-7.58 (m, $5 \mathrm{H}), 7.74(\mathrm{~d}, J=8.1 \mathrm{~Hz}, 2 \mathrm{H})$, 7.91-7.97 (m, 4 H), 8.09 (s, 2 H), 8.71 (s, $1 \mathrm{H}), 9.46$ (s, $1 \mathrm{H}, \mathrm{NH}), 9.87$ (s, $1 \mathrm{H}, \mathrm{NH})$.

${ }^{13} \mathrm{C}$ NMR $\left(50 \mathrm{MHz}\right.$, DMSO- $\left.d_{6}\right): \delta=112.8,117.3$, $117.9,120.6,120.8,121.7,123.4,128.3,128.8,129.0$, $129.7,130.4,132.0,132.2,132.5,132.7,133.1,133.2$, 136.6, 138.1, 138.3, 141.1, 142.4, 148.8, 155.6, 158.6.

Anal. Calcd for $\mathrm{C}_{32} \mathrm{H}_{20} \mathrm{Cl}_{4} \mathrm{~N}_{4}$ : C, 63.81; $\mathrm{H}, 3.35 ; \mathrm{N}$, 9.30. Found: C, 63.35; H, 3.43; N, 9.09.

$N^{2}, N^{4}$-Bis(3-chlorophenyl)-6,8-di(naphthalen-2-yl)quinazoline-2,4-diamine (6g)

Yellow powder; yield $85 \%(0.15 \mathrm{~g}) ; \mathrm{mp} 137^{\circ} \mathrm{C}$

${ }^{1} \mathrm{H}$ NMR $\left(200 \mathrm{MHz}\right.$, DMSO- $\left.d_{6}\right): \delta=6.80(\mathrm{~d}, J=7.0$ $\mathrm{Hz}, 1 \mathrm{H}), 6.98(\mathrm{t}, J=7.7 \mathrm{~Hz}, 1 \mathrm{H}), 7.22(\mathrm{~d}, J=7.4 \mathrm{~Hz}$, $1 \mathrm{H}), 7.44-7.57(\mathrm{~m}, 5 \mathrm{H}), 7.74(\mathrm{~d}, J=7.4 \mathrm{~Hz}, 1 \mathrm{H})$, 7.96-8.02 (m, $8 \mathrm{H}), 8.11-8.15(\mathrm{~m}, 3 \mathrm{H}), 8.26(\mathrm{~s}, 1 \mathrm{H})$, $8.33(\mathrm{~s}, 1 \mathrm{H}), 8.49(\mathrm{~s}, 1 \mathrm{H}), 8.93(\mathrm{~s}, 1 \mathrm{H}), 9.47(\mathrm{~s}, 1 \mathrm{H}$, $\mathrm{NH}), 9.99(\mathrm{~s}, 1 \mathrm{H}, \mathrm{NH})$.

${ }^{13} \mathrm{C}$ NMR $\left(50 \mathrm{MHz}, \mathrm{DMSO}-d_{6}\right): \delta=113.0,117.2$, $117.9,120.6,120.7,121.0,121.9,123.5,125.5,126.1$, $126.2,126.4,126.7,127.4,127.7,127.8,128.4,128.7$, $128.8,129.0,129.7,130.4,132.4,132.5,133.0,133.1$, 133.3, 133.6, 134.1, 136.9, 137.4, 138.0, 141.2, 142.4, 149.2, 155.6, 158.8 .

HRMS (ESI): $\mathrm{m} / \mathrm{z}[\mathrm{M}+\mathrm{H}]^{+}$Calcd for $\mathrm{C}_{40} \mathrm{H}_{26} \mathrm{Cl}_{2} \mathrm{~N}_{4}$ : 633.1607; found: 633.1606 .

\section{$N^{2}, N^{4}$-Bis(3-chlorophenyl)-6,8-bis(5-methyl- thiophen-2-yl)quinazoline-2,4-diamine (6h)}

Yellow powder; yield $76 \%(0.12 \mathrm{~g}) ; \mathrm{mp} 147^{\circ} \mathrm{C}$

${ }^{1} \mathrm{H}$ NMR (200 MHz, DMSO- $\left.d_{6}\right): \delta=2.50(\mathrm{~s}, 6 \mathrm{H}, 2$ $\left.\mathrm{CH}_{3}\right), 6.87(\mathrm{~s}, 2 \mathrm{H}), 6.97(\mathrm{~d}, J=8.6 \mathrm{~Hz}, 1 \mathrm{H}), 7.18$ 7.28 (m, $2 \mathrm{H}), 7.40-7.48(\mathrm{~m}, 2 \mathrm{H}), 7.59-7.63(\mathrm{~m}, 2 \mathrm{H})$, $7.92(\mathrm{~s}, 1 \mathrm{H}), 8.00(\mathrm{~d}, J=8.2 \mathrm{~Hz}, 1 \mathrm{H}), 8.10(\mathrm{~s}, 1 \mathrm{H})$, $8.24(\mathrm{~s}, 1 \mathrm{H}), 8.45$ (s, $1 \mathrm{H}), 9.38(\mathrm{~s}, 1 \mathrm{H}, \mathrm{NH}), 9.82$ (s, $1 \mathrm{H}, \mathrm{NH})$.

${ }^{13} \mathrm{C}$ NMR (50 MHz, DMSO- $\left.d_{6}\right): \delta=15.2\left(\mathrm{CH}_{3}\right), 15.3$ $\left(\mathrm{CH}_{3}\right), 113.0,117.7,118.2,119.2,121.2,121.3,122.1$, 123.6, 124.2, 125.4, 127.0, 128.3, 128.6, 129.9, 130.4, $133.1,133.2,136.7,139.4,140.5,141.0,141.4,142.0$, 147.3, 155.5, 158.7.

Anal. Calcd for $\mathrm{C}_{30} \mathrm{H}_{22} \mathrm{Cl}_{2} \mathrm{~N}_{4} \mathrm{~S}_{2}$ : C, 62.82; H, 3.87; N, 9.77. Found: C, $62.70 ; \mathrm{H}, 3.79 ; \mathrm{N}, 9.86$. 


\section{$N^{2}, N^{4}$-Bis(3-chlorophenyl)-6,8-di(pyridin-3-yl)-} quinazoline-2,4-diamine (6i)

Yellow powder; yield $80 \%(0.12 \mathrm{~g})$; mp $247{ }^{\circ} \mathrm{C}$

${ }^{1} \mathrm{H}$ NMR $\left(200 \mathrm{MHz}\right.$, DMSO- $\left.d_{6}\right): \delta=6.90(\mathrm{~d}, J=7.8$ $\mathrm{Hz}, 1 \mathrm{H}), 7.11-7.23$ (m, $2 \mathrm{H}), 7.42-7.58$ (m, $3 \mathrm{H}), 7.66$ $(\mathrm{d}, J=7.8 \mathrm{~Hz}, 1 \mathrm{H}), 7.95(\mathrm{~d}, J=8.9 \mathrm{~Hz}, 2 \mathrm{H}), 8.08-$ $8.23(\mathrm{~m}, 3 \mathrm{H}), 8.34(\mathrm{~d}, J=7.2 \mathrm{~Hz}, 1 \mathrm{H}), 8.63$ (bs, 2 $\mathrm{H}), 8.84(\mathrm{~s}, 1 \mathrm{H}), 8.91(\mathrm{~s}, 1 \mathrm{H}), 9.19(\mathrm{~s}, 1 \mathrm{H}), 9.50$ (s, $1 \mathrm{H}, \mathrm{NH}), 9.90(\mathrm{~s}, 1 \mathrm{H}, \mathrm{NH})$.

${ }^{13} \mathrm{C}$ NMR (50 MHz, DMSO- $\left.d_{6}\right): \delta=112.9,117.5$, $118.1,120.9,121.4,121.8,123.2,123.6,124.0,129.9$, $130.5,131.0,132.5,133.2,134.4,134.7,134.8,134.9$, 137.9, 141.0, 142.3, 148.1, 148.4, 148.7, 149.3, 150.4, 155.9, 158.7 .

HRMS (ESI): $\mathrm{m} / \mathrm{z}[\mathrm{M}+\mathrm{H}]^{+}$Calcd for $\mathrm{C}_{30} \mathrm{H}_{20} \mathrm{Cl}_{2} \mathrm{~N}_{6}$ : 535.1199; found: 535.1202 .

\section{$N^{2}, N^{4}$-Bis(3-chlorophenyl)-6,8-bis(3-nitrophenyl)-} quinazoline-2,4-diamine (6j)

Yellow powder; yield $71 \%(0.12 \mathrm{~g}) ; \mathrm{mp} 245^{\circ} \mathrm{C}$

${ }^{1} \mathrm{H}$ NMR $\left(200 \mathrm{MHz}\right.$, DMSO- $\left.d_{6}\right): \delta=6.86(\mathrm{~d}, J=7.2$ $\mathrm{Hz}, 1 \mathrm{H}), 7.10(\mathrm{t}, J=7.6 \mathrm{~Hz}, 1 \mathrm{H}), 7.22(\mathrm{~d}, J=7.2 \mathrm{~Hz}$, $1 \mathrm{H}), 7.43-7.54(\mathrm{~m}, 2 \mathrm{H}), 7.82(\mathrm{t}, J=7.2 \mathrm{~Hz}, 2 \mathrm{H})$, $7.96(\mathrm{~s}, 1 \mathrm{H}), 8.05-8.11(\mathrm{~m}, 2 \mathrm{H}), 8.22-8.42(\mathrm{~m}, 5 \mathrm{H})$, $8.66(\mathrm{~s}, 1 \mathrm{H}), 8.74(\mathrm{~s}, 1 \mathrm{H}), 8.85(\mathrm{~s}, 1 \mathrm{H}), 9.54(\mathrm{~s}, 1 \mathrm{H}$, $\mathrm{NH}), 9.96$ ( $\mathrm{s}, 1 \mathrm{H}, \mathrm{NH})$.

${ }^{13} \mathrm{C}$ NMR (50 MHz, DMSO- $\left.d_{6}\right): \delta=113.0,117.4$, $117.8,120.9,121.1,121.4,122.0,122.3,122.4,123.7$, $125.4,129.7,129.9,130.5,130.6,131.7,132.8,133.1$, 133.7, 135.4, 137.0, 140.7, 140.9, 141.1, 142.2, 147.7, 148.8, 149.2, 156.1, 158.7 .

Anal. Calcd for $\mathrm{C}_{32} \mathrm{H}_{20} \mathrm{Cl}_{2} \mathrm{~N}_{6} \mathrm{O}_{4}: \mathrm{C}, 61.65 ; \mathrm{H}, 3.23 ; \mathrm{N}$, 13.48. Found: C, 61.14; H, 3.35; N, 13.39 .

\section{$N^{2}, N^{4}$-Bis(3-chlorophenyl)-6,8-bis[3-(trifluoro-} methyl)phenyl]quinazoline-2,4-diamine (6k)

Yellow powder; yield $75 \%(0.14 \mathrm{~g})$; mp $141{ }^{\circ} \mathrm{C}$

${ }^{1} \mathrm{H}$ NMR $\left(200 \mathrm{MHz}, \mathrm{DMSO}-d_{6}\right): \delta=6.89(\mathrm{~d}, J=7.5$ $\mathrm{Hz}, 1 \mathrm{H}), 7.10(\mathrm{~d}, J=8.1 \mathrm{~Hz}, 1 \mathrm{H}), 7.16-7.23(\mathrm{~m}, 1$ H), $7.43(\mathrm{~d}, J=8.1 \mathrm{~Hz}, 1 \mathrm{H}), 7.51(\mathrm{~d}, J=9.0 \mathrm{~Hz}, 1$ H), 7.81-7.88 (m, $4 \mathrm{H})$, 7.93-7.95 (m, $3 \mathrm{H}), 8.10-8.15$ (m, $5 \mathrm{H}), 8.82(\mathrm{~s}, 1 \mathrm{H}), 9.50(\mathrm{~s}, 1 \mathrm{H}, \mathrm{NH}), 9.90(\mathrm{~s}, 1$ $\mathrm{H}, \mathrm{NH})$.

${ }^{13} \mathrm{C}$ NMR (50 MHz, DMSO- $\left.d_{6}\right): \delta=112.9,117.4$, 118.0, 120.9, 121.8, 123.6, $124.7(\mathrm{q}, J=271.9 \mathrm{~Hz})$, $124.8(\mathrm{q}, J=270.5 \mathrm{~Hz}), 125.2(\mathrm{q}, J=3.7 \mathrm{~Hz}),$, $(\mathrm{q}, J=3.7 \mathrm{~Hz}), 127.8,127.9(\mathrm{q}, J=31.5 \mathrm{~Hz}), 128.0$ $(\mathrm{q}, J=32.0 \mathrm{~Hz}), 129.7,130.5,131.1,132.4,132.6$, $133.2,133.3,136.5,141.0,142.3,143.4(\mathrm{q}, J=1.1$ $\mathrm{Hz}), 143.6$ (q, $J=1.1 \mathrm{~Hz}), 149.3,156.0,158.7$.

Anal. Calcd for $\mathrm{C}_{34} \mathrm{H}_{20} \mathrm{Cl}_{2} \mathrm{~F}_{6} \mathrm{~N}_{4}: \mathrm{C}, 61.00 ; \mathrm{H}, 3.01 ; \mathrm{N}$, 8.37. Found: C, 61.33; H, 3.03; N, 8.23.

\section{Synthesis of compounds 6l-t}

A solution of 6,8-dibromo-2,4-dichloroquinazoline 4 $(0.1 \mathrm{~g}, 0.28 \mathrm{mmol})$, amine $(1.12 \mathrm{mmol}, 4$ equiv) in a DME/EtOH mixture $(9 / 1,5 \mathrm{~mL})$ was heated under microwave irradiation (cf temperature in Table 3).
After cooling, 4-fluorophenylboronic acid $(86 \mathrm{mg}$, $0.62 \mathrm{mmol}, 2.2$ equiv), $\mathrm{PdCl}_{2}\left(\mathrm{PPh}_{3}\right)_{2}$ (10 mg, 14 $\mu$ mol, 0.05 equiv $), \mathrm{K}_{2} \mathrm{CO}_{3}(0.23 \mathrm{~g}, 1.68 \mathrm{mmol}, 6$ equiv) and water $(0.5 \mathrm{~mL})$ were introduced under argon. The mixture was heated at $100{ }^{\circ} \mathrm{C}$ under microwave irradiation for $1 \mathrm{~h}$. After cooling, $80 \mathrm{~mL}$ of water were added and the solution was extracted with dichloromethane $(3 \times 60 \mathrm{~mL})$. The organic layer was washed with water $(3 \times 100 \mathrm{~mL})$, dried over $\mathrm{Na}_{2} \mathrm{SO}_{4}$ and evaporated. The crude product was purified by column chromatography (silica gel, petroleum ether/ethyl acetate (9/1), (8/2 for $\mathbf{6 r}$ and $\mathbf{6 s}))$ and recrystallized from acetonitrile.

\section{6,8-Bis(4-fluorophenyl)- $N^{2}, N^{4}$-diphenyl- quinazoline-2,4-diamine (61)}

Brown powder; yield $71 \%(0.1 \mathrm{~g})$; mp $114{ }^{\circ} \mathrm{C}$

${ }^{1} \mathrm{H}$ NMR $\left(200 \mathrm{MHz}\right.$, DMSO- $\left.d_{6}\right): \delta=6.88(\mathrm{t}, J=6.7$ $\mathrm{Hz}, 1 \mathrm{H}), 7.10-7.16(\mathrm{~m}, 3 \mathrm{H}), 7.35-7.48(\mathrm{~m}, 6 \mathrm{H})$, 7.82-7.97 (m, $9 \mathrm{H}), 8.70(\mathrm{~s}, 1 \mathrm{H}), 9.15(\mathrm{~s}, 1 \mathrm{H}, \mathrm{NH})$, 9.77 (s, $1 \mathrm{H}, \mathrm{NH})$.

${ }^{13} \mathrm{C}$ NMR $\left(50 \mathrm{MHz}, \mathrm{DMSO}-d_{6}\right): \delta=112.8,114.6(\mathrm{~d}, J$ $=20.9 \mathrm{~Hz}), 115.9(\mathrm{~d}, J=21.2 \mathrm{~Hz}), 119.0,120.4$, $121.0,122.6,123.7,128.3,128.7,129.0(\mathrm{~d}, J=8.1$ $\mathrm{Hz}), 132.0,132.6(\mathrm{~d}, J=7.3 \mathrm{~Hz}), 135.8(\mathrm{~d}, J=3.3$ $\mathrm{Hz}), 136.2(\mathrm{~d}, J=3.3 \mathrm{~Hz}), 136.4,139.7,141.1,148.9$, 156.0, 158.8, $161.8(\mathrm{~d}, J=243.7 \mathrm{~Hz}), 162.0(\mathrm{~d}, J=$ $244.8 \mathrm{~Hz})$.

HRMS (ESI): $\mathrm{m} / \mathrm{z}[\mathrm{M}+\mathrm{H}]^{+}$Calcd for $\mathrm{C}_{32} \mathrm{H}_{22} \mathrm{~F}_{2} \mathrm{~N}_{4}$ : 501.1885; found: 501.1884 .

$N^{2}, N^{4}, 6,8$-Tetrakis(4-fluorophenyl)quinazoline-2,4diamine $(6 \mathrm{~m})$

Brown powder; yield $93 \%(0.14 \mathrm{~g}) ; \mathrm{mp} 230{ }^{\circ} \mathrm{C}$

${ }^{1} \mathrm{H}$ NMR $\left(200 \mathrm{MHz}\right.$, DMSO- $\left.d_{6}\right): \delta=6.96(\mathrm{t}, J=8.8$ $\mathrm{Hz}, 2 \mathrm{H}), 7.22-7.39(\mathrm{~m}, 6 \mathrm{H}), 7.77-7.83(\mathrm{~m}, 4 \mathrm{H})$, 7.93-7.96 (m, $5 \mathrm{H}), 8.67$ (s, $1 \mathrm{H}), 9.18(\mathrm{~s}, 1 \mathrm{H}, \mathrm{NH})$, 9.80 (s, $1 \mathrm{H}, \mathrm{NH})$.

${ }^{13} \mathrm{C}$ NMR (50 MHz, DMSO- $\left.d_{6}\right): \delta=112.7,114.6(\mathrm{~d}, J$ $=21.2 \mathrm{~Hz}), 115.3(\mathrm{~d}, J=22.0 \mathrm{~Hz}), 115.9(\mathrm{~d}, J=21.2$ $\mathrm{Hz}), 120.3,120.5(\mathrm{~d}, J=7.3 \mathrm{~Hz}), 124.7(\mathrm{~d}, J=7.7$ $\mathrm{Hz}), 129.0(\mathrm{~d}, J=8.1 \mathrm{~Hz}), 132.1,132.5,132.6(\mathrm{~d}, J=$ $7.7 \mathrm{~Hz}), 135.7(\mathrm{~d}, J=3.3 \mathrm{~Hz}), 135.9(\mathrm{~d}, J=1.8 \mathrm{~Hz})$, $136.2(\mathrm{~d}, J=3.3 \mathrm{~Hz}), 136.4,137.4(\mathrm{~d}, J=1.8 \mathrm{~Hz})$, $148.8,155.9,157.1(\mathrm{~d}, J=245.5 \mathrm{~Hz}), 158.9,161.8(\mathrm{~d}$, $J=243.7 \mathrm{~Hz}), 162.0(\mathrm{~d}, J=244.4 \mathrm{~Hz}), 163.5(\mathrm{~d}, J=$ $243.0 \mathrm{~Hz})$.

HRMS (ESI): $\mathrm{m} / \mathrm{z}[\mathrm{M}+\mathrm{H}]^{+}$Calcd for $\mathrm{C}_{32} \mathrm{H}_{20} \mathrm{~F}_{4} \mathrm{~N}_{4}$ : 537.1697; found: 537.1698.

$N^{2}, N^{4}$-Bis(2,5-dimethoxyphenyl)-6,8-bis(4-fluorophenyl)quinazoline-2,4-diamine (6n)

Yellow powder; yield 76\% (0.13 g); mp $184{ }^{\circ} \mathrm{C}$

${ }^{1} \mathrm{H}$ NMR (200 MHz, DMSO- $\left.d_{6}\right): \delta=3.45$ (s, $3 \mathrm{H}$, $\left.\mathrm{OCH}_{3}\right), 3.76\left(\mathrm{~s}, 9 \mathrm{H}, 3 \mathrm{OCH}_{3}\right), 6.39(\mathrm{dd}, J=2.3 \mathrm{~Hz}, J$ $=8.6 \mathrm{~Hz}, 1 \mathrm{H}), 6.81-6.87(\mathrm{~m}, 2 \mathrm{H}), 7.09(\mathrm{~d}, J=8.6$ $\mathrm{Hz}, 1 \mathrm{H}), 7.23-7.39(\mathrm{~m}, 6 \mathrm{H}), 7.76-7.83(\mathrm{~m}, 2 \mathrm{H})$, 7.93-8.00 (m, $4 \mathrm{H}), 8.66(\mathrm{~s}, 1 \mathrm{H}, \mathrm{NH}), 9.57(\mathrm{~s}, 1 \mathrm{H}$, $\mathrm{NH})$. 
${ }^{13} \mathrm{C} \mathrm{NMR}\left(50 \mathrm{MHz}, \mathrm{DMSO}-d_{6}\right): \delta=55.3\left(\mathrm{OCH}_{3}\right)$, $55.6\left(\mathrm{OCH}_{3}\right), 56.2\left(\mathrm{OCH}_{3}\right), 56.3\left(\mathrm{OCH}_{3}\right), 104.6$, $106.1,110.6,111.5,112.9,113.1,113.6,115.0(\mathrm{~d}, J=$ $21.6 \mathrm{~Hz}), 115.9(\mathrm{~d}, J=21.2 \mathrm{~Hz}), 120.4,128.0,128.9$ $(\mathrm{d}, J=8.1 \mathrm{~Hz}), 129.9,132.2,132.3(\mathrm{~d}, J=8.4 \mathrm{~Hz})$, $132.8,135.6(\mathrm{~d}, J=3.3 \mathrm{~Hz}), 136.0(\mathrm{~d}, J=2.9 \mathrm{~Hz})$, 136.6, 141.8, 148.0, 148.5, 153.2, 153.6, 155.7, 159.9, $161.7(\mathrm{~d}, J=244.8 \mathrm{~Hz}), 162.1(\mathrm{~d}, J=244.8 \mathrm{~Hz})$.

Anal. Calcd for $\mathrm{C}_{36} \mathrm{H}_{30} \mathrm{~F}_{2} \mathrm{~N}_{4} \mathrm{O}_{4}$ : C, 69.67; H, 4.87; N, 9.03. Found: C, 69.96; H, 4.81; N, 8.88.

\section{6,8-Bis(4-fluorophenyl)- $N^{2}, N^{4}$-dimesityl- quinazoline-2,4-diamine (6o)}

Yellow powder; yield 87\% (0.14 g); mp $205{ }^{\circ} \mathrm{C}$

${ }^{1} \mathrm{H}$ NMR $\left(200 \mathrm{MHz}, \mathrm{DMSO}-d_{6}\right): \delta=2.01(\mathrm{~s}, 6 \mathrm{H}, 2$ $\left.\mathrm{CH}_{3}\right), 2.19$ (s, $\left.6 \mathrm{H}, 2 \mathrm{CH}_{3}\right), 2.25\left(\mathrm{~s}, 3 \mathrm{H}, \mathrm{CH}_{3}\right), 2.28$ (s, $\left.3 \mathrm{H}, \mathrm{CH}_{3}\right), 6.80(\mathrm{~s}, 2 \mathrm{H}), 6.90-6.96(\mathrm{~m}, 4 \mathrm{H}), 7.28-$ 7.35 (m, 2 H), 7.71 (bs, 2 H), 7.90 (bs, 4 H), 8.62 (s, 1 H, NH), 9.28 (s, $1 \mathrm{H}, \mathrm{NH})$.

${ }^{13} \mathrm{C}$ NMR (50 MHz, DMSO- $\left.d_{6}\right): \delta=18.3\left(2 \mathrm{CH}_{3}\right)$, $18.4\left(2 \mathrm{CH}_{3}\right), 20.7\left(\mathrm{CH}_{3}\right), 20.8\left(\mathrm{CH}_{3}\right), 112.0,113.8$ (d, $J=20.5 \mathrm{~Hz}), 115.8(\mathrm{~d}, J=20.5 \mathrm{~Hz}), 120.5,128.1$, $128.6(\mathrm{~d}, J=7.3 \mathrm{~Hz}), 128.7,131.1,132.2(\mathrm{~d}, J=7.7$ $\mathrm{Hz}), 133.9$, 134.1, 134.3, 135.0 (d, $J=2.6 \mathrm{~Hz}), 135.2$, $135.6,135.8,136.5(\mathrm{~d}, J=2.9 \mathrm{~Hz}), 149.1,158.7$, $160.3,161.2(\mathrm{~d}, J=243.7 \mathrm{~Hz}), 161.8(\mathrm{~d}, J=243.4$ $\mathrm{Hz})$.

Anal. Calcd for $\mathrm{C}_{38} \mathrm{H}_{34} \mathrm{~F}_{2} \mathrm{~N}_{4}$ : C, 78.06; H, 5.86; N, 9.58. Found: C, 78.02; H, 5.72; N, 9.32.

$N^{2}, N^{4}$-Bis(3-fluorophenyl)-6,8-bis(4-fluorophenyl)quinazoline-2,4-diamine (6p)

Yellow powder; yield 86\% (0.13 g); mp $125^{\circ} \mathrm{C}$

${ }^{1} \mathrm{H}$ NMR (200 MHz, DMSO- $\left.d_{6}\right): \delta=6.68-6.71(\mathrm{~m}, 1$ $\mathrm{H}), 6.98-7.01(\mathrm{~m}, 1 \mathrm{H}), 7.19(\mathrm{t}, J=6.9 \mathrm{~Hz}, 1 \mathrm{H}), 7.32-$ 7.42 (m, $6 \mathrm{H}), 7.79$ (bs, $3 \mathrm{H}), 7.97-8.00$ (m, $5 \mathrm{H}), 8.72$ (s, $1 \mathrm{H}), 9.53$ (s, $1 \mathrm{H}, \mathrm{NH}), 9.92$ (s, $1 \mathrm{H}, \mathrm{NH})$.

${ }^{13} \mathrm{C}$ NMR (50 MHz, DMSO- $\left.d_{6}\right): \delta=112.7,114.9(\mathrm{~d}, J$ $=22.0 \mathrm{~Hz}), 115.9(\mathrm{~d}, J=21.2 \mathrm{~Hz}), 117.9,120.4,129.2$ $(\mathrm{d}, J=8.1 \mathrm{~Hz}), 129.6(\mathrm{~d}, J=9.2 \mathrm{~Hz}), 130.1(\mathrm{~d}, J=9.5$ $\mathrm{Hz}), 132.4$ (d, $J=8.1 \mathrm{~Hz}), 133.3,135.5,136.0,136.7$, $141.4,142.7,148.6,155.4,158.7,161.9(\mathrm{~d}, J=244.1$ $\mathrm{Hz}), 162.1$ (d, $J=244.4 \mathrm{~Hz}), 162.4(\mathrm{~d}, J=241.1 \mathrm{~Hz})$, $162.5(\mathrm{~d}, J=240.1 \mathrm{~Hz})$.

Anal. Calcd for $\mathrm{C}_{32} \mathrm{H}_{20} \mathrm{~F}_{4} \mathrm{~N}_{4}: \mathrm{C}, 71.64 ; \mathrm{H}, 3.76 ; \mathrm{N}$, 10.44. Found: C, 71.55; H, 3.65; N, 10.30.

\section{$N^{2}, N^{4}$-Bis(3,4-dichlorophenyl)-6,8-bis(4-fluoro-} phenyl)quinazoline-2,4-diamine (6q)

Yellow powder; yield 73\% (0.13 g); mp $231{ }^{\circ} \mathrm{C}$

${ }^{1} \mathrm{H}$ NMR $\left(200 \mathrm{MHz}, \mathrm{DMSO}-d_{6}\right): \delta=7.30-7.38(\mathrm{~m}, 5$ H), 7.57-7.67 (m, $2 \mathrm{H})$, 7.72-7.78 (m, $2 \mathrm{H}), 7.91-7.99$ $(\mathrm{m}, 3 \mathrm{H}), 8.09(\mathrm{~d}, J=7.9 \mathrm{~Hz}, 1 \mathrm{H}), 8.18(\mathrm{~s}, 1 \mathrm{H}), 8.24$ (s, $1 \mathrm{H}), 8.66$ (s, $1 \mathrm{H}), 9.57(\mathrm{~s}, 1 \mathrm{H}, \mathrm{NH}), 9.96(\mathrm{~s}, 1 \mathrm{H}$, $\mathrm{NH})$.

${ }^{13} \mathrm{C}$ NMR (50 MHz, DMSO- $\left.d_{6}\right): \delta=112.7,115.3(\mathrm{~d}, J$ $=21.6 \mathrm{~Hz}), 115.9(\mathrm{~d}, J=21.6 \mathrm{~Hz}), 119.1,119.8$,
$120.4,122.7,123.8,1240,125.6,129.1$ (d, $J=8.1$ Hz), 129.6, 130.1, 130.5, 131.0, 131.1, 131.4, 132.2 $(\mathrm{d}, J=8.1 \mathrm{~Hz}), 132.8(\mathrm{~d}, J=3.3 \mathrm{~Hz}), 133.6,135.0$, $135.8(\mathrm{~d}, J=2.6 \mathrm{~Hz}), 139.4,140.6,154.8,158.8$, $162.0(\mathrm{~d}, J=244.1 \mathrm{~Hz}), 162.2(\mathrm{~d}, J=244.8 \mathrm{~Hz})$.

HRMS (ESI): $\mathrm{m} / \mathrm{z}[\mathrm{M}+\mathrm{H}]^{+}$Calcd for $\mathrm{C}_{32} \mathrm{H}_{18} \mathrm{Cl}_{4} \mathrm{~F}_{2} \mathrm{~N}_{4}$ : 639.0302; found: 639.0302.

\section{$N^{2}, N^{4}$-Dicyclopentyl-6,8-bis(4-fluorophenyl)- quinazoline-2,4-diamine (6r)}

Yellow powder; yield 95\% (0.13 g); $\mathrm{mp} 242{ }^{\circ} \mathrm{C}$

${ }^{1} \mathrm{H}$ NMR $\left(200 \mathrm{MHz} \mathrm{CDCl}_{3}\right): \delta=1.65-1.85(\mathrm{~m}, 10 \mathrm{H}$, $\left.5 \mathrm{CH}_{2}\right), 2.02-2.14\left(\mathrm{~m}, 6 \mathrm{H}, 3 \mathrm{CH}_{2}\right), 4.30-4.37(\mathrm{~m}, 1 \mathrm{H}$, $\mathrm{CH}), 4.60-4.69$ (m, 1 H, CH), 6.90 (bs, $3 \mathrm{H}), 7.17-$ $7.23(\mathrm{~m}, 2 \mathrm{H}), 7.48-7.61(\mathrm{~m}, 3 \mathrm{H}), 8.74(\mathrm{~s}, 1 \mathrm{H}), 9.00$ (s, $1 \mathrm{H}), 9.24$ (s, $1 \mathrm{H}, \mathrm{NH}), 10.58$ (s, $1 \mathrm{H}, \mathrm{NH})$.

${ }^{13} \mathrm{C} \mathrm{NMR}\left(50 \mathrm{MHz}, \mathrm{CDCl}_{3}\right): \delta=23.9\left(2 \mathrm{CH}_{2}\right), 24.3(2$ $\left.\mathrm{CH}_{2}\right), 32.1\left(2 \mathrm{CH}_{2}\right), 32.8\left(2 \mathrm{CH}_{2}\right), 53.5(\mathrm{CH}), 54.7$ $(\mathrm{CH}), 111.0,115.4(\mathrm{~d}, J=21.6 \mathrm{~Hz}), 116.3(\mathrm{~d}, J=22.0$ $\mathrm{Hz}), 122.8,128.9$ (d, $J=8.1 \mathrm{~Hz}), 129.3,131.0$ (d, $J=$ $2.2 \mathrm{~Hz}), 131.3(\mathrm{~d}, J=8.1 \mathrm{~Hz}), 134.3,134.6$ (d, $J=2.6$ $\mathrm{Hz}), 135.3,152.7,159.5,162.4(\mathrm{~d}, J=247.4 \mathrm{~Hz})$, 162.7 (d, $J=248.5 \mathrm{~Hz})$.

HRMS (ESI): $\mathrm{m} / \mathrm{z}[\mathrm{M}+\mathrm{H}]^{+}$Calcd for $\mathrm{C}_{30} \mathrm{H}_{30} \mathrm{~F}_{2} \mathrm{~N}_{4}$ : 485.2511; found: 485.2511 .

\section{$N^{2}, N^{4}$-Dibenzyl-6,8-bis(4-fluorophenyl)- quinazoline-2,4-diamine (6s)}

Yellow powder; yield 88\% (0.13 g); mp $165^{\circ} \mathrm{C}$

${ }^{1} \mathrm{H}$ NMR (200 MHz, $\left.\mathrm{CDCl}_{3}\right): \delta=4.60\left(\mathrm{~s}, 2 \mathrm{H}, \mathrm{CH}_{2}\right)$, $4.81\left(\mathrm{~d}, J=4.6 \mathrm{~Hz}, 2 \mathrm{H}, \mathrm{CH}_{2}\right), 7.00-7.07(\mathrm{~m}, 5 \mathrm{H})$, 7.26-7.38 (m, $10 \mathrm{H}), 7.52-7.59$ (m, $5 \mathrm{H}), 7.72$ (s, $2 \mathrm{H})$.

${ }^{13} \mathrm{C}$ NMR $\left(50 \mathrm{MHz}, \mathrm{CDCl}_{3}\right): \delta=45.2\left(\mathrm{CH}_{2}\right), 45.3$ $\left(\mathrm{CH}_{2}\right), 111.6,114.5(\mathrm{~d}, J=21.2 \mathrm{~Hz}), 115.7(\mathrm{~d}, J=$ $21.6 \mathrm{~Hz}), 118.3,126.8,127.5,127.8(\mathrm{~d}, J=21.9 \mathrm{~Hz})$, 128.0, 128.3, 128.5 (d, $J=8.4 \mathrm{~Hz}), 128.6,132.0$ (d, $J$ $=7.8 \mathrm{~Hz}), 132.5(\mathrm{~d}, J=9.2 \mathrm{~Hz}), 134.9,136.1(\mathrm{~d}, J=$ $2.6 \mathrm{~Hz}), 136.6,138.4,138.5,140.1,160.3,160.4$, $162.2(\mathrm{~d}, J=245.9 \mathrm{~Hz}), 162.3(\mathrm{~d}, J=246.6 \mathrm{~Hz})$.

HRMS (ESI): $\mathrm{m} / \mathrm{z}[\mathrm{M}+\mathrm{H}]^{+}$Calcd for $\mathrm{C}_{34} \mathrm{H}_{26} \mathrm{~F}_{2} \mathrm{~N}_{4}$ : 529.2198; found: 529.2197.

\section{$N^{2}, N^{2}, N^{4}, N^{4}$-Tetraethyl-6,8-bis(4-fluorophenyl)- quinazoline-2,4-diamine (6t)}

Yellow oil; yield 85\% (0.11 g).

${ }^{1} \mathrm{H}$ NMR $\left(200 \mathrm{MHz}, \mathrm{CDCl}_{3}\right): \delta=1.17(\mathrm{t}, J=7.0 \mathrm{~Hz}, 6$ $\left.\mathrm{H}, 2 \mathrm{CH}_{3}\right), 1.39\left(\mathrm{t}, J=7.0 \mathrm{~Hz}, 6 \mathrm{H}, 2 \mathrm{CH}_{3}\right), 3.61(\mathrm{q}, J$ $\left.=6.7 \mathrm{~Hz}, 4 \mathrm{H}, 2 \mathrm{CH}_{2}\right), 3.68(\mathrm{q}, J=6.9 \mathrm{~Hz}, 4 \mathrm{H}, 2$ $\left.\mathrm{CH}_{2}\right)$, 7.07-7.19 (m, $\left.4 \mathrm{H}\right), 7.55-7.62(\mathrm{~m}, 2 \mathrm{H}), 7.74-$ $7.81(\mathrm{~m}, 3 \mathrm{H}), 7.87(\mathrm{~d}, J=2.1 \mathrm{~Hz}, 1 \mathrm{H})$.

${ }^{13} \mathrm{C} \mathrm{NMR}\left(50 \mathrm{MHz}, \mathrm{CDCl}_{3}\right): \delta=13.2\left(2 \mathrm{CH}_{3}\right), 13.6(2$ $\left.\mathrm{CH}_{3}\right), 41.8\left(2 \mathrm{CH}_{2}\right), 45.2\left(2 \mathrm{CH}_{2}\right), 112.3,114.1(\mathrm{~d}, J=$ $22.3 \mathrm{~Hz}), 115.6(\mathrm{~d}, J=21.2 \mathrm{~Hz}), 122.6,128.1(\mathrm{~d}, J=$ $7.7 \mathrm{~Hz}), 130.1,131.2,132.0$ (d, $J=7.7 \mathrm{~Hz}), 135.9$ (d, $J=3.2 \mathrm{~Hz}), 136.0,137.4(\mathrm{~d}, J=2.6 \mathrm{~Hz}), 151.8,157.3$, $161.9(\mathrm{~d}, J=245.2 \mathrm{~Hz}), 162.4(\mathrm{~d}, J=242.6 \mathrm{~Hz})$. 
HRMS (ESI): $\mathrm{m} / \mathrm{z}[\mathrm{M}+\mathrm{H}]^{+}$Calcd for $\mathrm{C}_{28} \mathrm{H}_{30} \mathrm{~F}_{2} \mathrm{~N}_{4}$ : 461.2511; found: 461.2511.

\section{References}

(1) (a) Miyaura, N.; Suzuki, A. Chem. Rev. 1995, 95, 2457. (b) Suzuki, A. Organomet. Chem. 1999, 576, 147. (c) Kotha, S.; Lahiri, K.; Kashinath, D. Tetrahedron 2002, 58, 9633. (d) Bellina, F.; Carpita, A.; Rossi, R. Synthesis 2004, 2419. (e) Phan, N. T. S.; Van Der Sluys, M.; Jones, C. W. Adv. Synth. Catal. 2006, 348, 609.

(2) Kirchhoff, J. H.; Netherton, M. R.; Hills, I. D.; Fu, G. C. J. Am. Chem. Soc. 2002, 124, 13662.

(3) (a) Nicolaou, K. C.; Montagnon, T.; Snyder, S. A. Chem. Commun. 2003, 551. (b) Padwa, A. Pure Appl. Chem. 2004, 76, 1933. (c) Wu, G.; Yin, W.; Shen, H. C.; Huang, Y. Green Chem. 2012, 41, 580. (d) Gembus, V.; Bonfanti, J.-F.; Querolle, O.; Jubault, P.; Levacher, V.; Hoarau, C. Org. Lett. 2012, 14, 6012.

(4) (a) Crozet, M. P.; Archaimbault, G.; Vanelle, P.; Nouguier, R. Tetrahedron Lett. 1985, 26, 5133. (b) Roubaud, C. ; Vanelle, P. ; Maldonado, J.; Crozet, M. P. Tetrahedron 1995, 51, 9643. (c) Amiri-Attou, O.; Terme, T.; Vanelle, P. Molecules 2005, 10, 545. (d) Castera-Ducros, C.; Paloque, L.; Verhaeghe, P.; Casanova, M.; Cantelli, C.; Hutter, S.; Tanguy, F.; Laget, M.; Remusat, V.; Cohen, A.; Crozet, M. D.; Rathelot, P.; Azas, N.; Vanelle, P. Bioorg. Med. Chem. 2013, 21, 7155 .

(5) (a) Kung, P.-P.; Casper, M. D.; Cook, K. L.; WilsonLingardo, L.; Risen, L. M.; Vickers, T. A.; Ranken, R.; Blyn, L. B.; Wyatt, J. R.; Dan Cook, P.; Ecker, D. J. J. Med, Chem. 1999, 42, 4705. (b) Jantova, S.; Spirkova, K.; Stankovsky, S.; Duchonova, P. Folia Microbiol. 1999, 44, 187. (c) Grovera, G.; Kini, S. G. Eur. J. Med. Chem. 2006, 41, 256.

(6) (a) Chien, T.-C.; Chen, C.-S.; Yu, F.-H.; Chern, J.-W. Chem. Pharm. Bull. 2004, 52, 1422. (b) Herget, T.;

Freitag, M.; Morbitzer, M.; Kupfer, R.; Stamminger, T.; Marschall, M. Antimicrob. Agents Chemother. 2004, 48, 4154.

(7) (a) Kunes, J.; Bazant, J.; Pour, M.; Waisser, K.; Slosarek, M.; Janota, J. Farmaco 2000, 55, 725. (b) Waisser, K.; Gregor, J.; Dostal, H.; Kunes, J.; Kubicova, L.; klimesova, V.; Kaustova, J. Farmaco 2001, 56, 803.

(8) (a) Doyle, L. A.; Ross, D. D. Oncogene 2003, 22, 7340. (b) Henderson, E. A.; Bavetsias, V.; Theti, D. S.; Wilson, S. C.; Clauss, R.; Jackman, A. L. Bioorg. Med. Chem. 2006, 14, 5020.

(9) (a) Rewcastle, G. W.; Palmer, B. D.; Bridges, A. J.; Hollis Showalter, H. D.; Sun, L.; Nelson, J.; McMichael, A.; Kraker, A. J.; Fry, D. W.; Denny, W. A. J. Med. Chem. 1996, 39, 918. (b) VanBrocklin, H. F.; Lim, J. K.; Coffing, S. L.; Hom, D. L.; Negash, K.; Ono, M. Y.; Gilmore, J. L.; Bryant, I.; Riese II, D. J. J. Med. Chem. 2005, 48, 7445. (c) Cope, H.; Mutter, R.; Heal, W.; Pascoe, C.; Brown, P.; Pratt, S.; Chen, B. Eur. J. Med. Chem. 2006, 41, 1124. (d) Verhaeghe, P.; Azas, N.; Hutter, S.; Castera-Ducros, C.; Laget, M.; Dumètre, A.; Gasquet, M.; Reboul, J.-P.; Rault, S.; Rathelot, P.; Vanelle, P. Bioorg. Med. Chem. 2009, 17, 4313. (e) Kabri, Y.; Azas, N.; Dumètre, A.; Hutter, S.; Laget, M.; Verhaeghe, P.; Gellis, A.; Vanelle, P. Eur. J. Med. Chem. 2010, 45,616 .

(10) (a) Gautheron Chapoulaud, V.; Audoux, J.; Plé, N.; Turck, A.; Quéguiner. G. Tetrahedron Lett. 1999, 40, 9005. (b) Connolly, D. J.; Lacey, P. M.; McCarthy, M.; Saunders, C. P.; Carroll, A.-M.; Goddard, R.; Guiry, P. J. J. Org. Chem. 2004, 69, 6572. (c) Verhaeghe, P.; Azas,
N.; Gasquet, M.; Hutter, S.; Ducros, C.; Laget, M.; Rault, S.; Rathelot, P.; Vanelle, P. Bioorg. Med. Chem. Lett. 2008, 18, 396. (d) Kabri, Y.; Gellis, A.; Vanelle, P. Eur. J. Org. Chem. 2009, 4059.

(11) (a) Mangalagiu, I.; Benneche, T.; Undheim, K. Acta Chem. Scand. 1996, 50, 914. (b) Mangalagiu, I.; Benneche, T.; Undheim, K. Tetrahedron Lett. 1996, 37, 1309. (c) Wipf, P.; George, K. M. Synlett 2010, 644.

(12) (a) Kabri, Y.; Crozet, M. D.; Szabo, R.; Vanelle, P. Synthesis 2011, 3115. (b) Kabri, Y.; Crozet, M. D.; Primas, N.; Vanelle, P. Eur. J. Org. Chem. 2012, 5595.

(13) (a) Rahaim Jr., R. J.; Maleczka Jr., R. E. Tetrahedron Lett. 2002, 43, 8823. (b) Cellier, P. P.; Spindler, J.-F.; Taillefer, M.; Cristau, H.-J. Tetrahedron Lett. 2003, 44, 7191. (c) Handy, S. T.; Bregman, H.; Lewis, J.; Zhang, X.; Zhang, Y. Tetrahedron Lett. 2003, 44, 427. (d) Navarro, O.; Marion, N.; Oonishi, Y.; Kelly III, R. A.; Nolan, S. P. J. Org. Chem. 2006, 71, 685. (e) Zawisza, A. M.; Muzart, J. Tetrahedron Lett. 2007, 48, 6738. (f) Zeng, M.; Du, Y.; Shao, L.; Qi, C.; Zhang, X.-M. J. Org. Chem. 2010, 75, 2556.

(14) Nakhai, A.; Stensland, B.; Svensson, P. H.; Bergman, J. Eur. J. Org. Chem. 2010, 6588.

(15) Zabeer, A.; Bhagat, A.; Gupta, O. P.; Singh, G. D.; Youssouf, M. S.; Dhar, K. L.; Suri, O. P.; Suri, K. A.; Satti, N. K.; Gupta, B. D.; Qazi, G. N. Eur. J. Med. Chem. 2006, 41, 429.

(16) Sheibley, F. E. J. Org. Chem. 1947, 12, 743.

(17) Volzhina, O. N.; Azimov, V. A.; Medvedev, B. A.; Kazakov, A. A.; Zhikhareva, G. P.; Bondarenko, V. A.; Yuzhakov, S. D.; Dolgun, O. V.; Mashkovskii, M. D.; Yakhontov, L. N. Khim.-Farm. ž. 1987, 21, 802. 
